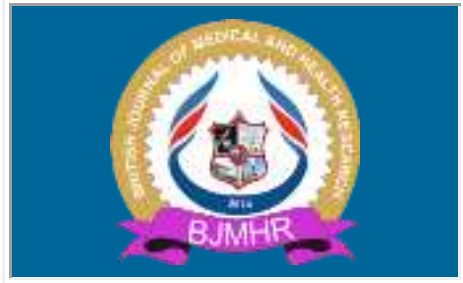

\title{
BJMHR
}

British Journal of Medical and Health Research

Journal home page: www.bjmhr.com

\section{Rare Presentations of Enteric Duplication Cysts.}

\section{Tanvi Luthra ${ }^{1}$, Santosh Kumar Singh*2, Meena Harsh ${ }^{4}$, Aisha $\mathrm{Naaz}^{3}$}

1. Himalayan Institute of Medical Sciences, SRHU, Dehradun

\begin{abstract}
Duplication cysts of the alimentary canal are rare congenital anomalies which can be present in any part of the GIT, from oral cavity till the anal canal. The clinical presentation depends on the location of the cyst. A large number of such lesions can be diagnosed prenatally using ultrasonography. The main treatment modality for enteric duplication cysts is surgery. Most commonly, these present with pain in abdomen, bleeding, and sometimes with obstruction, including intussusception. However, a significant number of these lesions are diagnosed incidentally. Here we are presenting a series of 3 cases showing the rare presentations of duplication cysts.
\end{abstract}

Keywords: Duplication cyst; congenital anomaly; intraluminal; pediatric 


\section{INTRODUCTION}

Duplication cysts of the alimentary canal are rare congenital malformations. These can involve any part of the GIT, from oral cavity till the anal canal. Most of these can be picked up during the antenatal period by USG. The presentation is protean depending on the location of the cyst. Most commonly, these present with pain in abdomen, bleeding, and sometimes with obstruction, including intussusception. However, a significant number of these lesions are diagnosed incidentally. We are reporting a series of 3 cases of duplication cysts with rare presentations.

\section{Case 1}

A 1-year-old female child presented with abdominal distension and pain in the abdomen since 3 days. There was history of constipation but no vomiting. She was a product of nonconsanguineous marriage. Antenatal USG was not done and perinatal history was not contributory.

Ultrasound of the abdomen showed distended gaseous bowel loops with mild free intraperitoneal fluid and a cystic lesion in the right side of abdomen.

CECT abdomen showed a well-defined non-enhancing hypodense lesion of near fluid density in the ileo-caecal region and adjoining part of caecum measuring $4.7 \times 3.2 \mathrm{~cm}$ (Fig1a). Exploration revealed a hypertrophied and grossly dilated distal ileum (Fig1b).

A sizeable firm mass was felt within the lumen of the terminal most ileum adjoining the caecum. An enterotomy showed a thick walled tense cyst arising from the bowel wall on the mesenteric side (Fig1c). The lesion was obstructing the ileo-caecal junction almost completely. The cyst had clear fluid.

The cyst was opened and the inner surface showed mucosal folds. The cyst wall was excised leaving a rim close to the parent bowel wall. The edges were over sewn with absorbable sutures. The patient recovered well after the procedure. Histopathological examination was consistent with a duplication cyst (Fig1d).
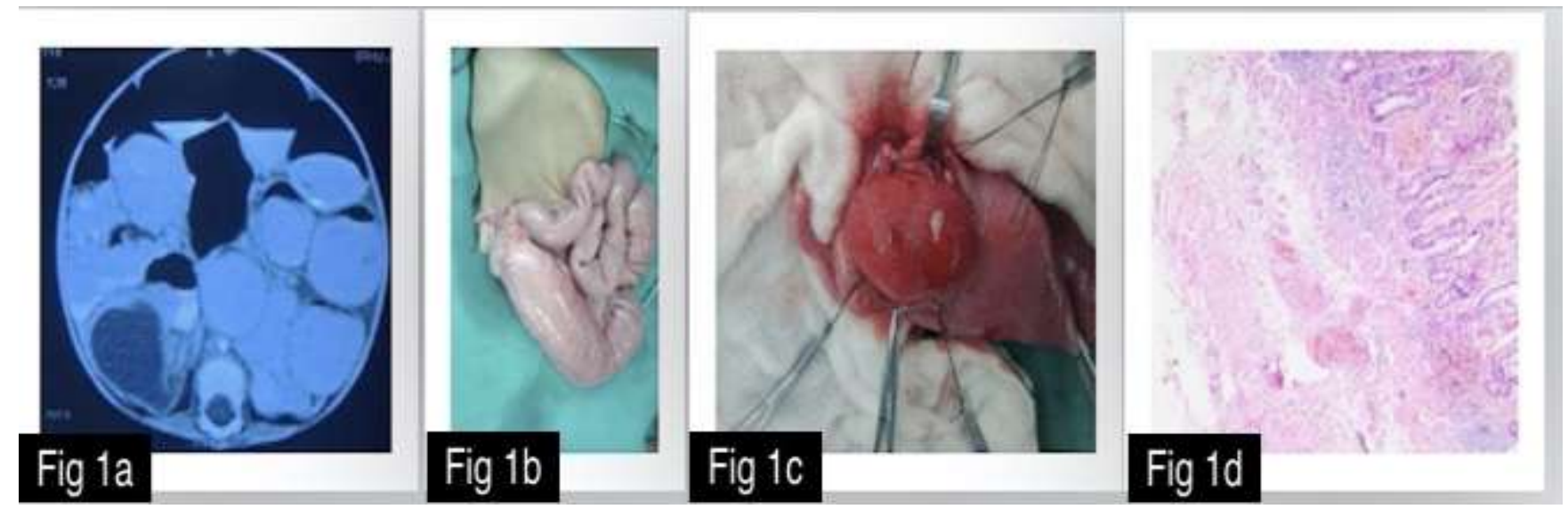

Figure 1a: Fluid filled lesion in ileo-caecal region 


\section{Figure 1b: Hypertrophied and dilated ileum}

\section{Figure 1c: Intraluminal duplication cyst}

Figure 1d: Intact lining epithelium with villi lined by columnar epithelium. Lamina shows mild chronic inflammatory infiltrate. Serosa shows mild oedema and hyperemia

Case 2

A 4-year-old female child was brought with c/o discharging sinus over left upper abdomen. There was history of a small swelling in the left upper abdomen a few weeks back which had spontaneously burst open and ever since it was discharging foul smelling brownish thin fluid (Fig2a).

Ultrasound abdomen revealed a large cystic lesion, approx. $7 x 5.3 \mathrm{~cm}$ in size adjacent to ileal loop, suggestive of a duplication cyst. The cyst was communicating with the sinus tract.

On exploration, a large cyst was found along the mesenteric border of the ileum with dense adhesions all around and was communicating with the opening on the anterior abdominal wall (Fig 2b). The cyst was not communicating with the lumen of the bowel. A resection and anastomosis of the involved segment of the ileum was done along with the sinus tract. The child recovered well after the procedure. Histopathological examination confirmed the diagnosis of enteric duplication cyst.
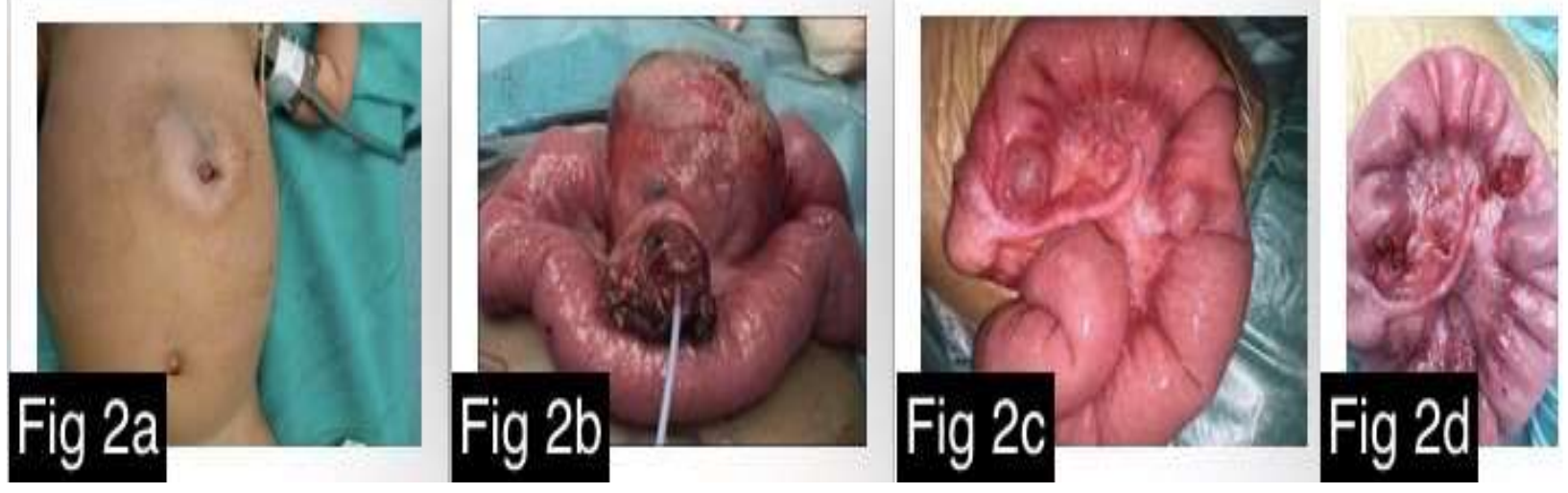

Figure 2a: Abdominal wall opening with discharge and local excoriation

Figure 2b: Ruptured ileal duplication cyst. Feeding tube seen in the cyst

Figure 2c: Cystic lesions in ileum

Figure 2d: After marsupialisation

\section{Case 3}

A 7 year old male child presented with complaints of on and off colicky pain in abdomen since 1 year. There were no other complaints. Child was thriving well. There was no significant past history either. Ultrasound of the abdomen showed 2 distinct cystic lesions in the central part of abdomen adjacent to the bowel loops. The nature of the lesions could not be ascertained.

In view of the recurrent definite symptoms, exploration was done which revealed 2 cystic lesions (each approximately $2 \mathrm{~cm}$ in diameter) along the mesenteric border of ileum (Fig2c). 
One was 1 foot proximal to IC junction and the other was just at the ileocaecal junction. Both cysts were not communicating with the bowel lumen.

The cysts were opened, mucosa excised and marsupialisation was done for both the cysts to avoid a large resection and to preserve the ileocaecal junction (Fig 2d).Histopathological examination confirmed the diagnosis of duplication cyst. The child recovered well and is doing well on 6 months follow up.

\section{DISCUSSION}

Duplications of the alimentary canal were first reported by Calder in 1733 and later, were described in detail by Ladd. He described the congenital lesions as those having a welldeveloped coat of smooth muscle, epithelial lining of intestinal mucosa and an anatomical association with a part of the gut. Duplication cysts have been reported in 1:10,000 live births and 1:4,500 autopsies (1) and may be present at any site, from oropharynx to anus. They have been noted in $0.2 \%$ of all children with a $2: 1$ male predominance. Ileum is the most common site of duplication cysts (33\%), followed by oesophagus (20\%), colon (13\%), jejunum (10\%), stomach $(7 \%)$ and duodenum (5\%). They may be contained within the lumen or may be extrinsic. Approximately $80 \%$ of the duplication cysts are cystic with the remaining $20 \%$ being of a tubular variety. The presentation would depend on the location of the cyst. Duodenal cysts can present with intussusception, melaena, abdominal pain, pancreatitis or GI bleeding from ulceration of the ectopic gastric mucosa. Jejunal duplications may cause bloating, constipation, volvulus and partial obstruction of the small bowel. Ileal cysts may be asymptomatic, or may present with abdominal pain, small bowel obstruction, a palpable abdominal mass, or hematochezia. Malignant transformation is rarely reported, but can occur in the setting of gastric mucosa heterotopia within the duplication cyst.

Prenatal sonographic diagnosis of duplications can be made as early as 12 weeks of gestational age. Abdominal ultrasound remains the investigation of choice for diagnosis of enteric duplication cysts and is only limited in the evaluation of oesophageal cysts. The classical findings on an ultrasound include presence of a cystic lesion with a double wall or a ring sign, caused by an inner hyperechoic mucosa and an outer hypoechoic smooth muscle layer. Being a dynamic study, ultrasonography also allows us to observe the peristalsis of the cyst wall. The usual presenting scenario in case of duplication cysts includes an intermittent history of constipation, due to partial bowel obstruction which may be overlooked. Most of the cysts are found attached to the mesenteric side of the bowel and are extra luminal. In the first case of our series, the cyst was intra luminal, fairly large in size and adjacent to the ileocaecal junction, and therefore presented with near complete obstruction so early in life.

Cases of perforated duplication cysts presenting as an acute abdomen (2) and infected 
duplication cysts (3) have been reported in literature, however the second case of the series shows a rare presentation of a ruptured cyst with discharging sinus which, to the best of our knowledge, has not been reported in literature yet.

Another rare presentation that we found was that of multiple cysts which have also been reported in other studies which found synchronous thoracic and abdominal duplications (4) and multiple enteric duplications which were identified antenatal in a twin fetus (5).

\section{CONCLUSION}

Due to the varied clinical features and often a nonspecific presentation, most cases of duplication cysts are not diagnosed early and can present at a later age with devastating complications. A curious eye during regular level II ultrasound will allow prenatal diagnosis and hence early and effective management of such cysts. The rarity of such lesions should not prevent us from keeping enteric duplication cysts as a plausible differential diagnosis in patients presenting with partial or complete intestinal obstruction to avoid a delay in the diagnosis and timely management of these lesions. Other rare entities may be found in cases of duplication cysts which must be kept in mind.

\section{REFERENCES}

1. Puligandla PS, Nguyen LT, St-Vil D, Flageole H, Bensoussan AL, Nguyen V-H, et al. Gastrointestinal duplications. J Pediatr Surg. 2003 May 1;38(5):740-4.

2. Elkadi TTH. Perforated duplication cyst in the ileum presented with acute abdomen. $\mathbf{J}$ Pediatr Surg Case Rep. 2015 Aug 1;3(8):341-3.

3. Zitteren LM van, Ruppert M, Beeck BO de, Wojciechowski M. Infected enteric duplication cyst. Case Rep. 2017 Sep 21;2017:bcr-2017-222391.

4. Kumar K, Dhull VS, Karunanithi S, Chakraborty PS, Roy SG, Ghosh S, et al. Synchronous thoracic and abdominal enteric duplication cysts: Accurate detection with 99mTc-pertechnetate scintigraphy. Indian J Nucl Med IJNM Off J Soc Nucl Med India. 2015;30(1):59-61.

5. Sheik S, Mathew M, Abdellatif M, Qureshi A, Mandhan P. Multiple Enteric Duplication Cysts in a Twin Fetus. Sultan Qaboos Univ Med J. 2013 Nov;13(4):5936.

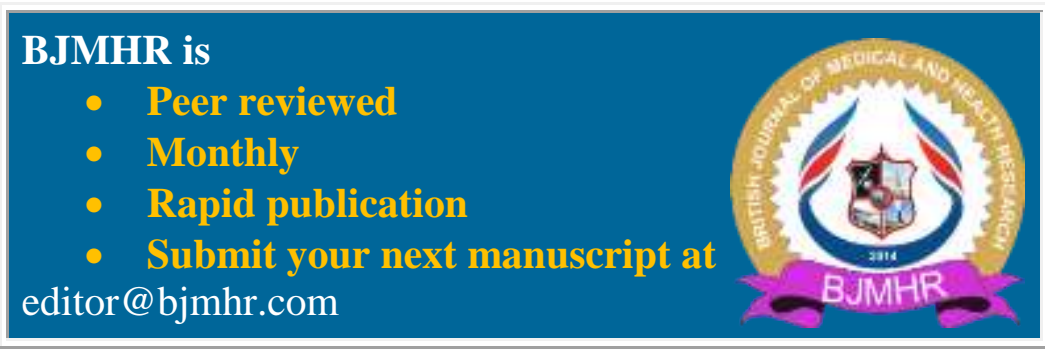

